\title{
Non-Compliance among Tuberculosis Patients on Self- Administration of Drugs in Ogbomoso Local Government Area of Oyo State. Nigeria.
}

\author{
Adeyeye James Oke ${ }^{1}$, Adewale Adegboyega $\mathrm{Oke}^{2}$ \\ ${ }^{1,}$ Department of Medical Microbiology, Faculty of Basic Clinical Sciences, Bowen University Teaching \\ Hospital, Ogbomoso. Nigeria. \\ 2, Department of Biological Sciences, College Of Natural Sciences, Redeemer's University, Mowe. Nigeria.
}

\begin{abstract}
This study was carried out to investigate non-compliance with medicaments prescriptions among tuberculosis patients on self-administration of multi-drug regimen in a local government of Oyo State, Nigeria. The isonicotinic acid method used is based on detecting the isoniazid metabolites-isonicotinic acid and isonicotinylglycine. The sensitivity of the method is high that reliably positive result is obtainable up to more than 24 hours after the ingestion of 100mg isoniazid. Three hundred (300) urine samples were collected from non-smoking tuberculosis (TB) patients on medicaments that included isoniazid: Fifty (50) samples from patients who took their drugs under supervision daily at the clinic; Two hundred and fifty (250) samples from patients who were given drugs away home for self-administration daily. One hundred percent (100\%) and Eighty two percent (82\%) showed isoniazid metabolites in their urines for supervised and self-administration respectively. Eighteen percent (18\%) on self-administration showed no isoniazid metabolites in their urines, indicating that they had not taken drugs in about 48 hours or more.
\end{abstract}

Keywords: Isoniazid, isonicotinic acid, isonicotinylglycine, Non-compliance, self-administration.

\section{Introduction}

Tuberculosis (TB) is among the leading causes of death worldwide. The World Health Organization (WHO) estimates that 32\% of the World population is infected with Mycobacterium tuberculosis, the causative agent of TB. (1-3). There were an estimated 9.2 millions new TB cases and 1.7 million deaths from TB in 2006 (4). Developed countries had shown downward trends of mortality and morbidity for many years, but in 1980s, morbidity plateaued or increased in areas and population groups with a high prevalence of HIV infection. It is much higher among the poor than the rich and usually higher in cities than in the rural areas. In 1989, the reported incidence of clinical disease in the USA was 10/100,000 population. Epidemics have been reported among persons congregated in enclosed spaces, such as Nursing homes, shelters for the homeless, hospitals, prisons and office buildings. The incidence of infection in developed countries has declined rapidly in recent decades: in the USA, the annual risk of new infection is estimated to average about 18/100,000 persons or less (5).

Tuberculosis constitutes a major health problem in Nigeria. In addition, the social stigma associated with the disease further compounds the problem. It has been estimated that about 250,000 new $\mathrm{Tb}$ cases occur yearly. Cases of $\mathrm{Tb}$ have been on the increase. In 1991 confronted with the increasing problems of $\mathrm{Tb}$ and Leprosy in Nigeria, the Federal Ministry of Health launched the National Tb and Leprosy Control programme. The Federal Government later invited developmental partners notably, WHO, Canadian International Development Agency (CIDA), International Federation of Anti-leprosy Association (ILEP).United States agency for International Development (USAID) (6). Damien Foundation Belgium (DFB), a NGO, is actively involved in the Tuberculosis and Leprosy Control programme in Oyo and Osun States of Nigeria, with the burdens of the provision of diagnostic reagents, equipments, man-power training and supply of anti-TB and antileprosy drugs.

Control of $\mathrm{Tb}$ remains one of the most challenges to global health. In 2005, there were an estimated 8.8 million new cases and 1.6 million deaths (1). TB is predominantly a disease of poverty with over $80 \%$ cases occurring in Asia and Africa. Although the greatest numbers live in highly populous countries of Asia, the highest incidence of disease is found in the WHO region of Africa. Nine countries in sub-Saharan Africa have recently reported estimated annual incidences in excess of 600 cases per 100,000 (4), a burden of disease not witnessed since before the advent of chemotherapy. The continued rise of $\mathrm{Tb}$ in this region may be largely attributable to AIDS pandemic combined with weak health care delivery systems. A new and potentially devastating threat to TB control is the emergency of strains that cannot be cured by standard anti-tuberculosis drug regimens. Drug resistant tuberculosis commonly arises through the selection of mutated strains by inadequate chemotherapy (7). 
Resistant to at least the two major anti tuberculosis drugs, isoniazid and rifampicin has been termed multidrug-resistant tuberculosis (MDR-TB). MDR-TB is increasing globally, especially in countries of former Soviet Union, Israel and the Republic of China (8-11).

Although the incidence of TB is steadily declining in Germany, numbers of cases with MDR-TB strains are increasing. In 2006, of 3501 TB cases in Germany for which resistance data were available, 78(2.2\%) were MDR-TB (12). These cases mainly occurred among immigrants from countries with high prevalence of MDRTB. $(12,13)$.

Studies carried out in some countries, (South Africa, USA., Germany, Kenya, India), have demonstrated that drug regimens that are highly successful in the treatment of TB in controlled clinical trials often fail when used routinely in mass treatment because patients fail to self-administer their drugs regularly, $(14,15,16)$. In the last 3 years $3 \%$ treatment failure has been recorded among patients on multidrug in the Oyo State DFB TB control programme. Focus has always been on emergence of mutated strains of Mycobacterium, fake and expired drugs, etc, with little attention to the patients' factor -the non-compliance factor. The objective of this study is to investigate the rate of non-compliance among the patients on self-administration of multi antiTB drugs in a treatment Zone in Oyo State.

In the DFB TB control programme of Oyo State, sputa from suspected cases of pulmonary Tuberculosis (PTB) are sent to the Laboratory for the demonstration of Acid fast bacilli (AFB), using ZeihlNeelson (Z-N) staining technique. Diagnosed (DX) cases are put on multidrug: Rifampicillin (150mg), isoniazid (75mg), pyrazinamide (400mg), ethambutol $\mathrm{HCl}(275 \mathrm{mg})$. Doses calculated on body weight are administered under the supervision of the Clinic supervisor, daily for 2 months. Thereafter follow-up (FU) patients are given one month supply of drugs at a time for self-administration at home for further 6 months. Sputum tests are repeated at 2, 5 and 8 months of treatment. Patients with demonstrable tubercle bacilli in their sputum after many months of multi-drug are considered MDR-TB.

\subsection{Materials}

\section{Materials and Methods}

Acetate buffer, $\mathrm{pH}$ 5.0,

$10 \%$ aqueous potassium cyanide,

$10 \%$ aqueous chloramine $-\mathrm{T}, 1 \%$

Barbituric acid in acetone/water (1:1 by volume).

Thymol Crystals.

All reagents were of British Drug Houses (BDH) products.

\subsection{Methods}

Isonicotinic acid method described by Ellard et al. (17) was employed in this study. It is based on detecting the isoniazid metabolites - isonicotinic acid and isonicotinylglycine in urine. Urine samples from nonsmoking $\mathrm{Tb}$ patients were collected as follows:

50 samples were collected from Dx patients on supervised administration of anti-Tb, regimens; 250 samples were collected from FU patients on unsupervised (Self-administration) anti-Tb drugs regimens.

Urine samples $(2.0 \mathrm{mls})$ which had been preserved by addition of few crystals of thymol, were pipetted into glass tubes together with $0.8 \mathrm{mls} 4 \mathrm{~m} \mathrm{pH} 5.0$ acetate buffer, and reacted by the addition at 15 -second interval of $0.4 \mathrm{mls} 10 \%$ aqueous potassium cyanide, $0.4 \mathrm{mls} 10 \%$ aqueous chloramine-T, and $2.0 \mathrm{mls} 1 \%$ barbituric acid in acetone/water (1:1 by volume). The reagents were freshly prepared. Negative controls were urine samples from healthy persons who were not on anti-Tb drug, positive control were urine samples from $\mathrm{Tb}$ patients who had been on supervised anti-Tb drugs. The results of tests were read after 45 minutes. The tests were carried out in a fume cupboard, using automatic pipette. Smokers were excluded from this study to avoid false positive.

\section{Results}

The tests were read after 45 minutes. Positive isonicotinic acid tests were indicated by the development of blue colour, whereas no such colour development was observed in the negative test (Fig.1).

$50 / 50(100 \%)$ Dx urine samples on supervised intake of drugs showed positive. 205/250 (82\%) of FU urine samples on self-administration of drugs showed positive, and 45/250 (18\%) FU urine samples showed negative to isonicotinic acid test (Table 1). 


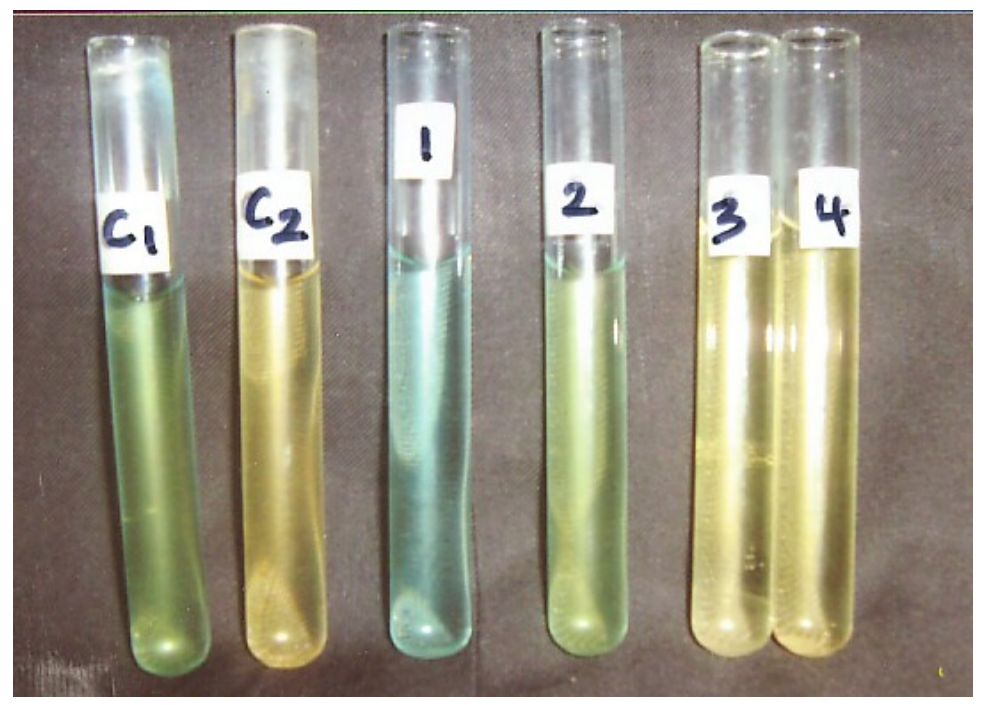

Figure 1: Showing positive and negative isonicotinic acid tests, and the controls.

C1: Positive control; C2: Negative control

$1 \& 2$ Positive tests; $3 \& 4$ Negative tests

Table 1: Numbers of positive and negative isonicotinic acid tests for supervised and self-administered anti-Tb regimens.

\begin{tabular}{lcc}
\hline & $\begin{array}{c}\text { Dx (supervised drug } \\
\text { intake) }\end{array}$ & FU (self administration of drug) \\
Number of tests & 50 & 250 \\
Number positive for isonicotinic acid test & $50(100 \%)$ & $205(82 \%)$ \\
Number negative for isonicotinic acid test & $0(0 \%)$ & $45(18 \%)$ \\
\hline
\end{tabular}

\section{Discussion}

Published reports from all over the World have shown that patients often fail to ingest the medicaments prescribed for them as hospital out-patients.

Non-compliance of self-administration may occur with drugs prescribed for a wide variety of conditions, for example, for the treatment of diabetes hypertension, tuberculosis, leprosy or for the prophylaxis of rheumatic fever, etc (18-21). Non-compliance or total abstinence can set in when symptoms of disease apparently subside, or when users get bored, especially when medicaments have to be in prolonged terms.

Several simple qualitative urine-test methods have been described to enable the ingestion of isoniazid, the most effective and widely used anti-tuberculosis drug, to be monitored (22). A recent investigation has demonstrated the suitability of using isoniazid as a maker to monitor the regularity of self-administration of other drugs and the sensitivity is such that reliably positive results are obtainable for only about 12 hours after the ingestion of isoniazid $(23,24)$.

The method used in this study is more sensitive for monitoring isoniazid ingestion which should enable it to be applied to urine samples collected from about one to 24 hours from patients on self-administration daily therapy doses of isoniazid substantially less than $200 \mathrm{mg}$. This method reliably detects concentration of $0.75 \mu \mathrm{g} / \mathrm{ml}$ isonicotinic acid and isonicotinylglycine in urine for more than 24hours after ingestion of isoniazid.

The results of this study showed $100 \%$ and $82 \%$ positivity among patients on daily supervised and selfadministration of medicaments respectively. $18 \%$ of self-administering group was negative (Table 1). This percentage is significant, and could contribute to MDR scourge.

Palenduz et.al. (2003) in their study obtained a remarkable percentage (33\%) of patients that were noncompliant with their treatment (25)

This showed that not all self-administering patients comply with medicament prescriptions. Further studies, however, need to be carried out to significantly relate non-compliance with MDR in this locality.

\section{Conclusion}

There was $82 \%$ compliance, and $18 \%$ non-compliance among the self-administering group in this study. Negative isonicotinic acid tests indicated that the patients had not taken their drugs in the past 48 hours or more since this test reliably detects concentrations of $0.75 \mu \mathrm{g} / \mathrm{ml}$ of metabolites in urine. Eighteen percent (18\%) non-compliance among self-administering group is significant. There is, therefore, the need for a strategy 
development to ensure strict compliance with medicaments among the tuberculosis patients to check treatment failure, and emergence of MDR strains of Mycobacterium tuberculosis in this locality.

The results obtained in this test proved the sensitivity and reliability of isonicotinic acid method for monitoring ingestion of isoniazid and that the use of urine colour test for monitoring drug adherence in tuberculosis treatment is not only reliable but quick, simple and inexpensive $(26,27,28,29)$.

\section{Acknowledgement}

We sincerely acknowledge Dr. S. O. Idowu, Faculty of Pharmacy, University of Ibadan who assisted in getting some of the reagents; Mrs. Adigun R., Chest Clinic, Baptist Medical Centre, Ogbomoso who assisted in the collection of samples from patients.

\section{References}

[1] World Health Organization. Anti-tuberculosis resistance in the World. Report No. 4. (Geneva: The organization, 2008).

[2] C. Monica, Medical Laboratory Manual for tropical countries Vol.II. (England: Stephen Austin and Sons Ltd. Hertford 1981).

[3] M. F Sydney and J. B . Ellen, Bailey \& Scott's Diagnostic Microbiology. (New York, USA: The CV Mosby co. 1986)

[4] N.R. Gandhi, A. Moll, A.W. Sturm, R. Pawinski, T. Govender, U. Lalloo , K. Zeller, J. Andrews and G. Friendland Extensively drug-resistant tuberculosis as a cause of death in patients co-infected with tuberculosis and HIV in a rural area of South Africa, Lancet, 368(9547), 2006, 1575-1580.

[5] S.B. Abram, Control of Communicable Diseases in Man (USA: American Public Health Association 1990)

[6] L. Eyitayo., National Tuberculosis and Leprosy control programme. Workers' Manual 4th ed 2004.

[7] B. Eker, J. Ortmann, G.B. Migliori, G. Sotgiu , R. Muetterlein, R. Centis , H. Hoffmann , D. Kirsten , T. Schaberg, S. RueschGerdes , C. Lange C and German TBNET Group, Multidrug- and extensively drug-resistant tuberculosis, Germany. Emerging Infectious Diseases, 14(11), 2008, 1700-1706.

[8] World Health Organization. Anti-tuberculosis drug resistance in the World. (Geneva: The Organization, 1997).

[9] World Health Organization. Global tuberculosis Control: Surveillance planning, financing (Geneva: The organization, 2007).

[10] W. Burman, D. Benator D, A. Vernon, A. Khan, B. Jones, C. Silva , C. Lahart, S. Weis, B. King, B. Mangura, M. Weiner and W. El-Sadr, Tuberculosis Trials Consortium. Acquired rifamycin resistance with twice-weekly treatment of HIV-related tuberculosis, American Journal of Respiratory and Critical Care Medicine, 173(3), 2006, 350-356.

[11] P.A. LoBue., and K.S. Moser, Isoniazid - and rifampicin - resistant tuberculosis in San Diego Country, California, United States 1993-2002. International Journal of Tuberculosis and Lung Disease, 9, 2005, 501-506

[12] O. Ratsirahonana, R.V.Rasolofo, T. Rasolonavalona, V. Rakotonirina, A. Rakotoarisaonina, A. Rakotoherisoa, M. Ralamboson ,B. Cauchoix, D.Rakotondramarina and H. Ramarokoto., Mycobacterium tuberculosis resistance to anti-tubercular agents in Antananarivo. 2000, Archives de Institut Pasteur de Madagascar, 68(1-2),2002, 44-47.

[13] F.M. Salaniponi, T.E. Nyirenda, J.R. Kemp, S.B. Squire, P. Godfrey-Faussett and A.D.Harries, Characteristics, management and outcome of patients with recurrent tuberculosis under routine programme conditions in Malawi. International Journal of Tuberculosis and Lung Disease, 7(10), 2003, 948-952.

[14] G.S. Azhar, DOTS for TB relapse in India: A systematic review, Lung India, 29(2), 2012, 147-153.

[15] U.Greinert and P. Zabel, Tuberculosis-current therapeutic principles, Internist (Berl).44(11), 2003, 1394-1405.

[16] P.W. Kent, W. Fox, A.B. Miller, A.J. Nunn, R. Tall, and D.A. Mitchison, The therapy of pulmonary tuberculosis in Kenya: a comparison of the results achieved in controlled clinical trials with those achieved by the routine treatment services. Tubercle,51(1), 1970, 24-38.

[17] G.A. Ellard and Greenfield, A sensitive urine-test method for monitoring the ingestion of isoniazid, Journal of Clinical Pathology, 30(1), 1977, 84-87.

[18] W. Fox, Self-administration of medicaments. A review of published work and a study of the problems, Bulletin of the International Union against Tuberculosis, 32, 1962, 307-331

[19] A.M.W. Porter, The problem of the self-administration of drugs. M.D. Thesis University of London, 1968.

[20] A.M. Porter, Drug defaulting in a general practice, British Medical Journal, 1(5638), 1969, 218-222.

[21] B. Blackwell, The drug defaulter, Clinical Pharmacology and Therapeutics, 13, 1972, 841-848.

[22] F.A. Sirgel, J.S. Maritz, A. Venter, G. Langdon, P.J. Smith and P.R. Donald, Monitoring the ingestion of anti-tuberculosis drugs by simple non-invasive methods, International Journal of Pharmaceutics, 307(2), 2006, 182-187.

[23] A. Mkopi, N. Range ,F. Lwilla, S. Egwaga, A. Schulze, E. Geubbels ,F. van Leth, Adherence to tuberculosis therapy among patients receiving home-based directly observed treatment: evidence from the United Republic of Tanzania, PLoS One, 7(12), 2012, e51828

[24] J.E. Stark, G.A. Ellard , P.T. Gammon and W. Fox, The use of isoniazid as a marker to monitor the self-administration of medicaments, British Journal of Clinical Pharmacology, 2(4), 1975, 355-358.

[25] A. Palanduz, D. Gültekin ,E. Erdem E, and N. Kayaalp Low level of compliance with tuberculosis treatment in children: monitoring by urine tests, Annals of Tropical Paediatrics, 23(1), 2003,47-50

[26] T. Eidlitz-Markus, A. Zeharia , G. Baum, M. Mimouni and J. Amir, Use of the urine color test to monitor compliance with isoniazid treatment of latent tuberculosis infection, Chest, 123(3), 2003,736-739.

[27] K.E. Schmitz, M.F .Hovell , C.A. Wong, N.J. Kelley, D. Nilsen ,E.J. Blumberg, L.L. Hill , C.L. Sipan, B. Kolody and D.A. Chatfield, The reliability and practicality of the Arkansas method assay of isoniazid adherence, Clinical Nursing Research, 19(2), 2010, 131-143.

[28] Y. Hanifa K. Mngadi, J. Lewis, K. Fielding, G. Churchyard and A.D.Grant, Evaluation of the Arkansas method of urine testing for isoniazid in South Africa, International Journal of Tuberculosis and Lung Disease.11(11), 2007,1232-1236.

[29] S. Perry, M.F. Hovell, E. Blumberg, J. Berg, A. Vera , C. Sipan, N. Kelley, K. Moser, A. Catanzaro and L. Friedman, Urine testing to monitor adherence to TB preventive therapy. Journal of Clinical Epidemiology,55(3), 2002, 235-238. 